\title{
Mechanical Conversion for High-Throughput TEM Sample Preparation
}

\author{
L. Zaykova-Feldman and T.M. Moore \\ Omniprobe, Inc., 10410 Miller Road, Dallas, TX 75238
}

The use of focused ion-beam (FIB) microscopes has become common for the preparation of specimens for later analysis in the transmission electron microscope (TEM). The "Total Release"TM in-situ lift-out method has been developed for high-throughput TEM sample preparation $[1,2]$. The process of in-situ lift-out can be simplified into three successive steps. The first is the excision of the wedge using focused ion-beam milling and extraction of the wedge from its trench. The second is the "holder-attach" step, during which the wedge is translated on the probe tip point to the TEM sample holder. Then it is attached to the TEM holder (typically with ion beam-induced metal deposition) and later detached from the probe tip point. The third and final step is the thinning of the wedge into an electron-transparent thin section using focused ion beam milling.

A significant portion of the total time involved in completing a TEM sample with in-situ lift-out method is spent during the transfer and attachment of TEM sample to a sample holder in the holder-attach step. The relative amount of time involved depends on the amount of time required to mechanically isolate the lift-out sample from the initial bulk sample (ion beam milling rate), but will vary between $30 \%$ to $60 \%$ of the total time for TEM sample preparation. However, direct mechanical conversion from lift-out sample to TEM sample holder eliminates the holder-attach step and provides several key throughput and resource advantages [3]. For example, the semiconductor wafer can be returned to the process flow immediately after lift-out. Thinning of the sample can be performed immediately, or later in an off-line FIB. The use of the off-line FIB for final thinning reduces the load on the critical in-line (clean room) FIB and reduces the level of FIB expertise required by process engineers who operate the in-line FIB.

The mechanical conversion is conducted using a mechanical press (shown in Figure 1) and one of a variety of TEM coupons. The press serves for cutting a TEM sample holder from a TEM coupon and joining a probe tip point with an attached TEM sample to the sample holder. This press can be located either inside or outside the vacuum chamber of the FIB or other analytical instrument. Being a mechanical procedure, this type of conversion is very reliable. It provides strong and robust bonding of the probe tip point and the TEM grid. Other advantages of the mechanical conversion include its low cost and speediness. A standard $3 \mathrm{~mm}$ diameter TEM grid is produced which is compatible with any conventional TEM holder.

There are two types of coupons used in mechanical conversion. The first type is designed for milling the TEM sample from the top-side (surface) and its central part remaining after cutting is shown in Figures 2 and 3. The second type, shown in Figure 4, is designed for back-side milling to avoid the "shower curtain" effect that may occur in topside thinning due to reduced milling speed at patterned dense materials at the device 
surface [4]. Both types of coupon have rectangular shape with the C-shaped hole in the middle as shown in Figure 1. One or more probe tips with TEM sample attached are placed on a coupon as shown in Figures 3 and 4 and be permanently attached to a coupon using the mechanical press. The system described here enables high throughput for inline process control and provides simple and robust solution for TEM sample preparation process.

[1] T.M. Moore, U.S. Patent 6570170.

[2] L.A. Gianuzzi et al., "FIB Lift-Out for Defect Analysis," in Analysis Techniques of Submicron Defects, 2002 Supplement to the EDFAS Failure Analysis Desktop Reference, ASM International, Materials Park, Ohio 29-35 (2002).

[3] Patent pending.

[4] S.M. Schwarz et al., Microsc. Microanal. 9 (Suppl. 2), (2002) 116-117.

[5] The authors wish to thank to G. Amador and R. Kruger for their dedicated efforts.

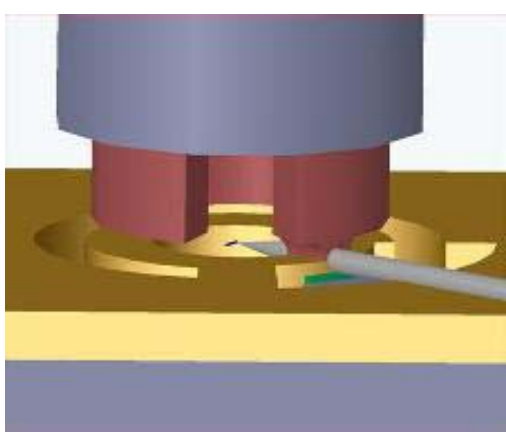

Fig.1. The press for mechanical conversion.

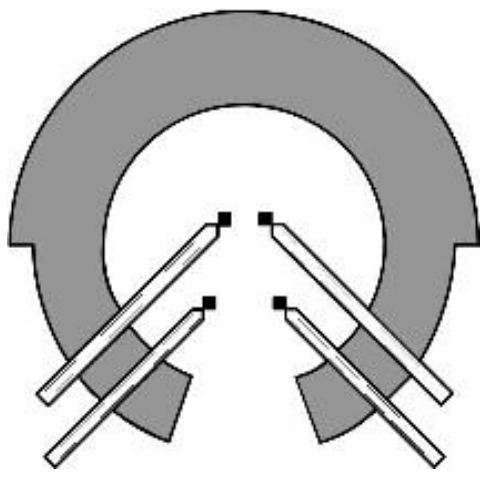

Fig.3. The coupon designed for top-side milling with four tips attached.

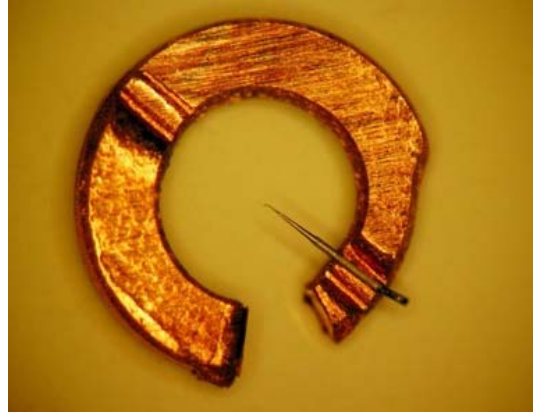

Fig.2. The coupon designed for topside milling with one tip attached.

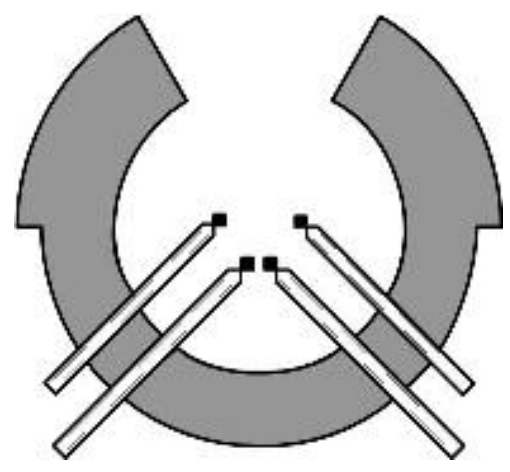

Fig.4. The coupon designed for back-side milling with four tips attached. 